\title{
Erratum: Transport coefficients for driven granular mixtures at low density [Phys. Rev. E 88, 052201 (2013)] and Heat flux of driven granular mixtures at low density: Stability analysis of the homogeneous steady state [Phys. Rev. E 97, 022902 (2018)]
}

\author{
Nagi Khalil and Vicente Garzó
}

(Received 7 April 2019; published 13 May 2019)

DOI: 10.1103/PhysRevE.99.059901

We have realized that the first-order contributions $T_{i}^{(1)}$ to the partial temperatures $T_{i}$ are also involved in the Chapman-Enskog solution to the first-order distributions $f_{i}^{(1)}$. The inclusion of the term $T_{i}^{(1)}$ changes some expressions displayed in Appendix B of our first paper. In a similar way to the expansion of the fluxes, the partial temperatures $T_{i}$ must be expanded as

$$
T_{i}=T_{i}^{(0)}+\epsilon T_{i}^{(1)}+\cdots .
$$

The existence of a nonzero first-order contribution $T_{i}^{(1)}$ induces a breakdown of the energy equipartition, additional to the one already present in the homogeneous steady state. Since $T_{i}^{(1)}$ is a scalar, it is coupled to $\boldsymbol{\nabla} \cdot \mathbf{U}$ and has the form

$$
T_{i}^{(1)}=\varpi \nabla \cdot \mathbf{U},
$$

where $\varpi$ depends on the hydrostatic pressure $p$ and the total temperature $T$ as well as the parameters of the thermostat and the mixture. The fact that the total temperature is not affected by the gradients implies necessarily $T_{2}^{(1)}=-n_{1} T_{1}^{(1)} / n_{2}=$ $-\left(n_{1} / n_{2}\right) \varpi \nabla \cdot \mathbf{U}$ and, hence, the hydrostatic pressure $p=n T=n_{1} T_{1}^{(0)}+n_{2} T_{2}^{(0)}$. This means that the pressure tensor $P_{\alpha \beta}^{(1)}$ is still given by Eq. (74) of our first paper. However, as mentioned before, some of the expressions of the first-order approximation change due to the presence of $T_{i}^{(1)}$. In particular, Eqs. (B3) and (B4) of the first paper should read

$$
\begin{gathered}
D_{t}^{(1)} p=-\frac{d+2}{d} p \nabla \cdot \mathbf{U}-2 \gamma_{\mathrm{b}} n \sum_{i=1}^{2} \frac{x_{i} T_{i}^{(1)}}{m_{i}^{\beta}}-p \zeta^{(1)}, \\
D_{t}^{(1)} T=-\frac{2}{d} T \nabla \cdot \mathbf{U}-2 \gamma_{\mathrm{b}} \sum_{i=1}^{2} \frac{x_{i} T_{i}^{(1)}}{m_{i}^{\beta}}-T \zeta^{(1)},
\end{gathered}
$$

where the constant $\beta$ is defined in Eq. (5) of our first paper. According to Eqs. (3) and (4), the expression (B14) of the first paper for the coefficient $E_{1}(\mathbf{V})$ should be changed to

$$
\begin{aligned}
E_{1}(\mathbf{V})= & \left(\frac{d+2}{d}+\zeta_{U}\right) p \frac{\partial f_{1}^{(0)}}{\partial p}+\left(\frac{2}{d}+\zeta_{U}\right) T \frac{\partial f_{1}^{(0)}}{\partial T} \\
& +2 \frac{\gamma_{\mathbf{b}}}{T} x_{1} \frac{m_{2}^{\beta}-m_{1}^{\beta}}{m_{1}^{\beta} m_{2}^{\beta}} \varpi\left(p \frac{\partial f_{1}^{(0)}}{\partial p}+T \frac{\partial f_{1}^{(0)}}{\partial T}\right) \\
& +\frac{1}{d} \mathbf{V} \cdot \frac{\partial f_{1}^{(0)}}{\partial \mathbf{V}} .
\end{aligned}
$$

Note that Eq. (5) agrees with Eq. (B14) of the first paper when $\beta=0$.

On the other hand, all the above changes do not affect the expressions of the transport coefficients associated with the fluxes of mass, momentum, and energy. In particular, the diffusion coefficients and the shear viscosity coefficient were determined in our first paper whereas the heat flux transport coefficients were computed in the second paper where a stability analysis of the homogeneous steady state was also carried out. Consequently, the relevant results obtained in the first and second papers remain unchanged when $T_{i}$ is replaced by its zeroth-order approximation $T_{i}^{(0)}$. The inclusion of $T_{i}^{(1)}$ only affects the calculation of the first-order contribution $\zeta_{U}$ to the cooling rate. However, given that the coefficient $\zeta_{U}$ was neglected (since its magnitude is expected to be very small) in the stability analysis performed in the second paper, then the conclusions of this paper remain valid. 